\title{
AWARENESS OF USAGE OF SUNSCREENS AMONG SCHOOL CHILDREN IN KANDY, SRI LANKA
}

\author{
N. G. P. D. NAWARATHNA, H. M. D. R. HERATH, D. B. M. WICKRAMARATHNE, M. H. F. SAKEENA, \\ C. B. GUNAWARDHANE, S. H. T. SUDESHIKA
}

Department of pharmacy, Faculty of Allied Health Sciences, University of Peradeniya

Email: priyankadamayanthi2@gmail.com

Received: 25 Mar 2016 Revised and Accepted: 08 Nov 2016

\begin{abstract}
Objective: The objective of this research was to evaluate the awareness of usage of sunscreens among school children in Kandy, Sri Lanka.

Methods: School children (138) who do outdoor sports were recruited from six schools. Knowledge and attitudes towards the usage and application of sunscreens were assessed using a researcher administered questionnaire. Statistical analyses were done using Statistical Package for the Social Science (SPSS version-16).
\end{abstract}

Results: Among the school children who do outdoor sports, 71 (51.4\%) of participants used sunscreens and 67 (48.6\%) of participants did not use sunscreens.

Conclusion: According to the results most of the school children have a lack of knowledge, attitudes and practice towards the usage of sunscreens.

Keywords: Sunscreens, UV, Knowledge, Attitudes, Application

(c) 2017 The Authors. Published by Innovare Academic Sciences Pvt Ltd. This is an open access article under the CC BY license (http://creativecommons.org/licenses/by/4. 0/) DOI: http://dx.doi.org/10.22159/ijpps.2017v9i1.11863

Ultra Violet (UV) radiation is composed of Ultra Violet A (UVA), Ultra Violet B (UVB) and Ultra Violet C (UVC). UVC radiation is completely absorbed before reach to the earth. Therefore, solar UV radiation in the earth surface composed a large amount of UVA radiation and a small amount of UVB radiations [1]. During intentional sun exposure (ISE), significant portions of the trunk, shoulders, and of the upper parts of the limbs of humans are frequently uncovered. The most typical ISE behaviour is sunbathing. Non-intentional sun exposure (NISE) represents sun exposure during daily life activities. During NISE, the most exposed skin areas to the sun usually are the head, neck, hands, and forearms. Lifetime accumulated NISE is mainly associated with the occurrence of solar keratoses and squamous cell carcinoma (SCC) [2]. Sunburn is the acute reaction of the skin from the damage by UV exposure. According to the estimations, $90 \%$ of UV light reaching the earth is UVA which contributes to the visible signs of ageing and skin degeneration. The remaining $10 \%$ of UV light is UVB which responsible for sunburn erythema [3]. And also according to the measurements, UV irradiance increases by $6-8 \%$ per $1000 \mathrm{~m}$ increase in latitude. Also part of the UV radiation that reaches to the ground absorbed by the earth and part of it reflect the surface. Therefore the reflected radiation can be caused harm to the people who stay on the earth surface. The signs and symptoms of sunburn include erythema, edema, blisters, ulcerations, and pain [3] Prevention of overexposure is especially important during childhood and adolescence because overexposure to UV radiation is the major cause for skin cancers [4].

Sunscreens are topical preparations that can be used to reduce penetration of harmful solar UV wavelengths. Sunscreens should prevent acute and chronic actinic damage of sun exposure by delaying photo-aging and preventing carcinomas and melanoma induction [5].

The sun protection factor (SPF) is defined as the ratio of the least amount of UV energy required to produce a minimal erythema on skin protected by sunscreen to the amount of energy required to produce the same erythema on the unprotected skin [6].

Sunscreen preparations are generally assessed for their ability to protect against erythema formation [5]. Most of the cosmetic products that contain sunscreen chemicals offer various SPF levels up to $15-50$ or higher. A sunscreen with an SPF of 15 filters out approximately $94 \%$ of the UVB rays. One with a SPF of 30 filters out $97 \%$ of UVB. The SPF applies for UVB rays only. The protection provided against UVA rays in chemical sunscreens is about $10 \%$ of the UVB rating [5]

Sri Lanka is a country that closer to the equator, where UV levels are higher. According to the literature, even shorter periods of exposure can lead to skin disorders. As a result of UV exposure, there is a possibility to increase the risk of infection and reduce the efficiency of immunisation. The majority of Sri Lankans does not get skin cancers due to UV exposure because of dark skin but get photodermatitis and premature ageing due to excessive UV exposure. So the UV exposure is a serious global implication. The most vulnerable population for this is children living in countries located close to the equator like Sri Lanka. Therefore development and implementation of sun protection strategies should be essential for them. According to the World Health Organization's 2014 UV index reports, the UV index of Sri Lanka is in between 8-12 throughout the year. If UV index is high the risk of sunburn due to sun exposure is high. It is estimated that in the majority of person's lifetime, UV exposure occurs during childhood. Also, there are strong evidence that exposure to UV radiation is a risk factor for developing skin cancer later in their lives. Therefore, children who engaged in outdoor sports have a higher risk from UV rays than others. Even in the shade, indirect UV levels can be sufficiently high to be harmful. High levels of the skin pigment melanin appear to reduce the risk of common skin cancers for people with darker skin. But in the case of severe sun exposure, melanin appears to be less effective in protecting against melanoma [7]. When skin cancer occurs in people with darker skin, it is likely to be detected. Later in that time the cancer is more advanced and therefore more dangerous. Excessive exposure to UV radiation is an issue and affects all skin types equally.

In order to above mention facts, the general objective of this study was to evaluate the knowledge and attitudes towards the usage and application of sunscreens among school children who do outdoor sports.

The study was conducted among school children who were involving in outdoor sports like swimming, cricket, netball, basketball, hockey, etc. in Kandy, Sri Lanka. The sample size was 138 school children who are involved in above outdoor sports and their age was between 14-18 y. Skin type of the students was not 
considered, and it was a limitation factor for the study. Students were selected from three boys' schools and three girls' schools, and the male to female ratio was 1:1.

A researcher administered questionnaire was used to collect data from students after getting their verbal consent. The pre-test was done using $10 \%$ of the study population. Three children who are doing outdoor sports were taken for pre-test from each school.

The questionnaire was based on the knowledge and attitudes among the school children towards usage and application of the sunscreens. In addition to that, it was included questions about on demographic information. Age, gender, sport were considered as variables. Knowledge and attitude towards sunscreen usage were assessed through time duration of exposure to sun, experience about sunburn, method use to protect from sun rays, knowledge about the SPF, and the factors when buying a sunscreen. Application of sunscreens assessed through time and frequency of reapplication.

Statistical analyses were undertaken using Statistical Package for the Social Sciences (SPSS version-16) software programme. The results were presented either as means or percentage frequencies and $95 \%$ confidence intervals, as appropriate.

The permission was obtained from zonal education office in Kandy to recruit school children from schools for this study. Ethical approval was obtained by the ethical review committee in the Faculty of Allied Health Sciences, University of Peradeniya, Sri Lanka.

Total of 138 school children recruited from 6 schools in Kandy, Sri Lanka including 69 girls from 3 girls' schools and 69 boys from 3 boys' schools. Age of the school children is between 14 to $18 \mathrm{y}$. Those who engaged with cricket, hockey, netball, basketball, rugby and any other outdoor sports were recruited for the study. The relationship of gender and engaging sports is shown in fig. 1.

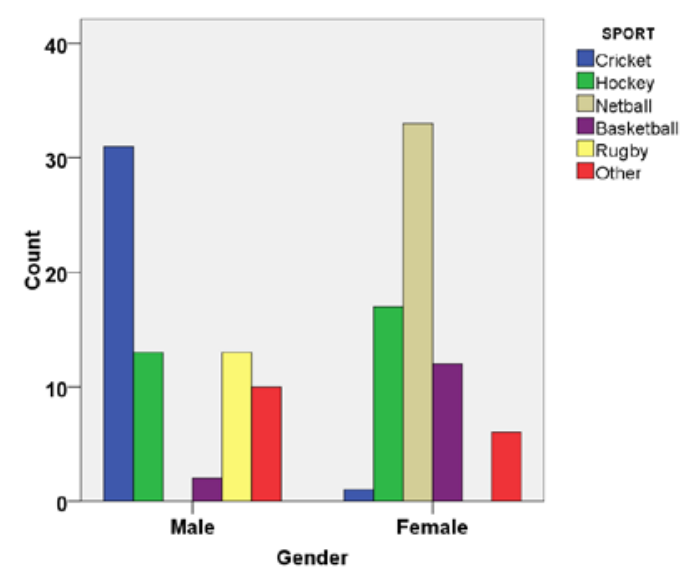

Fig. 1: The Relationship of gender and engaging sports

The maximum participation for outdoor sports activities was observed in age 14 and 15 . That represents $36(26.1 \%)$ participants from the whole sample. Cricket and netball were the two sports that school children are highly engaged in this study.32 (23.2\%) male participants were engaged in cricket and $33(23.9 \%)$ female participants were engaged in netball. Gender differences can be seen within the sports.

The majority, 137 of 138 (99.3\%) of the study population reported that they have exposed to the sun mostly during $2 \mathrm{p}$. m. to $4 \mathrm{p}$. m. $73.9 \%$ of the participants expose to the sun more than or slightly 3 $\mathrm{h}$ per day. $72(51.5 \%)$ participants have experienced the sunburn during last three months, and $66(47.8 \%)$ participants have never experienced sunburn. According to the results, girls have sunburn experience than boys.39 (56.5\%) of male students and 27 (39.1\%) female students have no experiences about the sun burn.56 (43.5\%) male students and $42(60.9 \%)$ female students have experienced about sunburn in last three months. It was also found that 72 (52.2\%) participants used any other methods to protect from the sun rays and $66(47.8 \%)$ participants not used any other methods to protect from sun rays. In the group of participants who used any other methods, 7 (5.1\%) participants were staying in the shade, 44 $(31.9 \%)$ were wearing a hat, and $24(17.4 \%)$ were wearing clothes covering most of the arms and legs.

Among the participants 53(38.4\%) had heard about the SPF and 85 (61.6\%) participants had never heard about SPF. Also, there were 18 participants never heard about SPF even though they used a sunscreen.

$57(41.3 \%)$ participants from the sample had considered the SPF value when purchasing a sunscreen. Only $14(10.1 \%)$ participants had considered the SPF value when purchasing sunscreen, and they think it is important. 62 (44.9\%) participants could not remember about SPF value of their sunscreen.

$35(24.5 \%)$ participants thought that the application of sunscreen help to increase the exposing time under sun rays without sun burning and $103(74.6 \%)$ of participants thought that there is no relationship between application of sunscreen and sun exposure.51.4\% (71) of participants were able to mention the health benefit of sunscreens. $86(62.3 \%)$ participants thought that expose to the sun can be affected to the serious health problems and $52(37.7 \%)$ participants mentioned that they never think there is a harmful effect from sun exposure and also they had no problem to expose to the sun. 132 (95.4\%) participants never participated to any of awareness programme about the sun protection behavior and harmful effects of sun exposure within their school life and $6(2.2 \%)$ of participants mentioned that they have watched television programmes related to UV radiation and protective methods.

Among the school children who do outdoor sports, 71 (51.4\%) participants used sunscreens and $67(48.6 \%)$ participants did not use sunscreens. Gender variation can be seen among the participants who use sunscreens. The usage of sunscreens among girls is higher than boys. Among the male participants only 32 $(45.7 \%)$ students use sunscreens and among the female participants only $39(56.5 \%)$ students use sunscreens.

The time of application of sunscreens also vary among the study population. $31(22.5 \%)$ participants always apply sunscreens before 30 min they expose to the sun. Among them 19 were females, and 12 were males. $29(21 \%)$ participants applied sunscreens at the same time they expose to the sun or before come to the school. $9(6.5 \%)$ participants always applied sunscreen during the game. Among them 4 were females, and 5 were males. 5 of them reapplied their sunscreens during tea break or lunch break. $9(6.5 \%)$ participants did reapplication only in the tournament days. Some of the participants thought that reapplication is not important and it is difficult to reapply during the game. 49 (35.5\%) participants never applied sunscreen during the game.

$35(25.4 \%)$ of participants always applied the same amount of sunscreen each day and each application. Among them, 19 participants are female students, and 16 participants are male students.23 (16.7\%) of participants never applied the same amount of sunscreen.

Some of them thought the application of sunscreen in cloudy days is meaningless.51 (37\%) participants never apply sunscreen in cloudy days. Only 7 (5.1\%) participants always apply sunscreen without considering whether it is a cloudy day or not. Among them, 5 are female participants and 2 are male participants. In all time $2.2 \%$ participants have experienced about photosensitivity reaction after applying sunscreen. Among them, 2 are male students and 1 is a female student. 55 (39.9\%) participants have not experienced about the photosensitivity reactions.

Skin cancers are within the first eighty causes of deaths according to the health profile of Sri Lanka and the world [8]. According to UV index, UV intensity levels on a scale of 1 to $11+$, where 1 indicates a low risk of overexposure and $11+$ signifies an extreme risk. UV index reports, the UV index of Sri Lanka is in between 8-12 throughout the year. If UV index is high the risk of sunburn due to sun exposure is 
high. So as Sri Lankans we have a high risk of getting sun burn due to sun exposure. According to the WHO recommendations, if UV index is higher than 2, application of sunscreen is recommended [9].

When considering the results of this study, 99.3\% ( $n=137)$ of school children expose to the sun during $2 \mathrm{p}$. m. to $4 \mathrm{p}$. m. time period. According to the literature, UV intensity level is high during the period of $11 \mathrm{a}$. m. to $3 \mathrm{p}$. m. Therefore, the majority of the school children expose to high UV intensity level in every day. Too much sun exposure is dangerous through the whole life cycle. The children should be highly protected from sun exposure because of the high sensitivity of their skin and cumulative effects of sunburn [1]. Therefore the school children who do outdoor sports have a high risk when they expose to the sun in the above mentioned time period. Also when increasing the time of sun exposure, the harmful effects on the skin can be increased. Ground reflection is also affected to that. That means the part of UV radiation that is reflected back to space also affected to get burn the skin.

Among the students who have not experienced about the burning sensation, they might have burns because sometimes UV radiation cannot be sensed by the humans due to the absorption in the outermost skin layers [1]. According to the results of this study, children use formulations like cream or emulsion, but none of them used other formulations such as a spray. The formulation of sunscreen can be an important factor to influence the individuals' willingness to use sunscreen [10]. Those who do not use sunscreens thought that application of sunscreen is not important and there is no any special benefit from the usage of sunscreens.

When comparing with the results of previous studies, various reasons such as having a skin that does not burn easily, already have protective tan, takes too much time to apply sunscreen, not outdoors enough to warrant use sunscreen, nuisancance and greasy to apply, feels hot and sweaty, expensive and forgotten to apply regularly for not using sunscreens have been mentioned [10].

To protect the skin from UV radiation wearing clothes to cover the whole body, applying sunscreens and staying in the shade are important [11]. The application of sunscreens is more important because the face, neck and some parts of hands and legs are not covered from clothes. Shade is the cheapest sun protection method, and it provides the protection from direct UV rays, but it cannot provide the protection from indirect UV rays. Due to heat and other uniform requirements, it is not practical for those engaging in open field activities to wear hats or long-sleeved shirts for protection from UV radiations. Therefore those children should apply sunscreens as the primary option of UV protection [11].

When evaluating the knowledge towards the usage of sunscreens among the school children, none of them knew the meaning of SPF even though they heard the term SPF. Some of them who used sunscreens have never seen the label of SPF on their sunscreen product. With reference to the results, the majority of students used sunscreens without knowing about the SPF. A previous study has revealed that sunscreens which have high SPF value help to get the extra protection but sunscreens which have SPF value greater than 30 not provide higher protection and that type of sunscreens are manufactured only for marketing purposes [1]. Therefore, considering the SPF value is important when purchasing a sunscreen.

The skin types and the UV index are also important factors that affect the SPF values of sunscreens. A sunscreen should be selected according to the factors mentioned above to get the maximum protection and avoid the unwanted effects from the sunscreen. There are six skin types. They are categorised from pale skin to dark skin [12]. The children who have the pale skin should use sunscreens which have high SPF when comparing with those who have darker skin. The students who used sunscreen mentioned that sunscreens are important to protect the skin from UV radiation, to reduce the darkening and to burn of the skin, etc. According to the results of previous studies, there are more benefits of usage of sunscreen other than prevent the sun burning [13]. They are; protection against skin cancers, perceiving them at high risk of skin cancer, protection against ageing and wrinkling, extending the time in the sun etc. [13]. But in this study, none of them was able to mention that type of benefits. Lack of knowledge about UV radiation and sunscreens can be the factors for that. And also among the participants who thought that sun exposure leads to serious health problems was not able to mention any serious health problem of UV radiation like skin cancers. The students who thought there is no any adverse effect of the sun rays mentioned that expose to the sun is necessary for vitamin $\mathrm{D}$ production in the body. But the time that exposes to the sun is needed for the vitamin D protection is less [1]. With reference to the results, inadequate participation to awareness programmes regarding sun protection can be the reason for their poor knowledge about the UV radiation. According to the guidelines of United States Food and Drug Administration (USFDA) the sunscreens that labelled as water resistant or sweat resistant are important to stay on the skin longer even if they get wet from water or sweat. Therefore consideration of water resistant or sweat resistant ability of sunscreen is also important for students who engaged sports like swimming or who are over sweating.

To get the better protection from UV radiation appropriate methods and time of application of the sunscreen are very important as well as the SPF value of the sunscreen which is matching for the skin type. Furthermore, daily use of sunscreen reduces the skin damage produced by UV exposure compared with the intermittent use of equal or higher SPF products [10]. Due to the cross-sensitivity reactions, performing a patch test by each individual is important prior to application of sunscreen. For that application of the small amount of sunscreen to the inner aspect of the forearm can be done [6]. According to the guidelines that described by the U. S FDA to get maximum benefit from sunscreen, it should be applied before 30 min. Then the product has time to soak into the skin. To obtain the adequate amount of sunscreen on the skin the first reapplication of sunscreen after $30 \mathrm{~min}$ is important [14]. Also to achieve $2 \mathrm{mg} / \mathrm{cm}^{2}$ concentration 1 ounce or $30 \mathrm{ml}$ should be applied [14]. Application of liberal amount of sunscreen on face, ears, nose, lips, back of neck, top of feet and areas of the head exposed by balding or thinning hair is important to get the maximum protection. After the first reapplication, reapply sunscreen two hourly is important to maintain the initial thickness of sunscreen leave on the skin.

According to the results, most of the girls apply sunscreens at home before come to the school. School rules and regulations are the reasons for that. However, the majority are not applying the sunscreen during the game. Among the children who applied sunscreens, some of them have done reapplication during the tea break and lunch break. Some of them mentioned that the disciplinary rules of tournaments also restrict reapplication of sunscreen. Furthermore, rules and regulations of schools affect for the poor attitudes towards the application of the sunscreens. The majority could not mention the exact frequency of reapplication of sunscreen after the first application.

Even though participants apply sunscreens in the face, neck and hands, no one apply sunscreens on ears, neck and feet. Some of them only apply the sunscreens on the face. All of them were unable to mention the amount of sunscreen that they apply. They mentioned that they did not like to apply a high amount of sunscreen due to leave a white film on the skin after application of sunscreen. Sunscreen containing mineral pigments can leave a white film on the skin and this can be affected to encourage the people to apply less than the required amount [10].

To get the protection from UV rays, plenty of sunscreen should be applied. Each application they should apply the same amount.

The most of the school children thought that in cloudy days there is no UV rays come to the ground and it is useless of application of sunscreen. In cloudy days $80 \%$ of UV rays reach to the ground. As other days there is a risk from UV rays for people who are exposing to the sun in cloudy days. Some of the students have experienced about photosensitivity reactions after applying of sunscreen.

Also, some children had photosensitivity reactions for some sunscreens available in the market. Therefore strengthening the regulations in the registration of sunscreens is important in Sri Lanka like tropical countries. Addition to the above, increasing the number of awareness programmes related to UV exposure and 
sunscreen application for school children, teachers in charge of sports and coachers will be beneficial in tropical countries which have high UVI even though the majority of people have dark skin type.

School children were engaged in their practices during peak sun hours without the protection of shade. Due to rules and regulations of schools and sports, they could not wear hats or long sleeved shirts for the protection of sun exposure. Therefore the primary option they have to protect from UV radiation is applying a sunscreen.

The knowledge and attitudes towards the usage of sunscreens among school children who do outdoor sports in Kandy zone is poor. The majority did not follow the proper method to apply and reapply sunscreen during the games. They are not aware of SPF value of their sunscreens. Therefore the improvement of awareness towards the usage and application of sunscreens among school children who do outdoor sports is important to reduce the unwanted effects and to get the maximum benefit.

\section{CONFLICTS OF INTERESTS}

Declared none

\section{REFERENCES}

1. Vanicek K, Frei T, Litynska Z, Schmalwieser A. UV index for the public, diabetes, heart disease and osteoporosis. Am J Clin Nutr 1998;79:362-71.

2. Autier P, Dore JF, Boniol M. Sunscreen use and increased duration of intentional sun exposure: still a burning issue. Int J Cancer 2007;121:1-5.

3. Miners AL. The diagnosis and emergency care of heat-related illness and sunburn in athletes: a retrospective case series. Journal of the Canadian Chiropractic Association; 2000.

4. Basch CH, Charles E, Baschand KV. High school student's use of sunscreen and Indoor tanning devices among a national representative; 2014. p. 6.

5. Liardet S, Scaletta C, Panizzon R, Hohlfeld P, Applegate LL. Protection against pyrimidine dimers, and 8-hydroxy-2'deoxyguanosine expression in the ultraviolet-irradiated human skin by sunscreen: difference between UVB+UVA and UVB alone sunscreen. J Investigate Dermatol 2001;117:1437-41.

6. Diffey B. Has the sun protection factor had its day. Br Med J 2000;320:176-7.

7. Riordan DLO, Kevin B, Lunde KB, Orschitz J, Glanz K. A noninvasive objective measure of sunscreen use and reapplication cancer epidemiology biomarkers and prevention, Cancer Prevention in Primary Care: Melanoma: prevention and early diagnosis; 1994. p. 308, 1682.

8. Health profile, Sri Lanka; 2011.
9. World Health Organization. Sun Protection and Schools How to make differences; 2003.

10. Phillips TJ, Bhawan J, Yaar M, Bello Y, Diffey DB. Has the sun protection factor had its day; 2000. p. 176-7.

11. Wickenheiser M, Baker MK, Gaber R, Blatt H, Robinson JK. Sun protection preferences and behaviours among young adult males during maximum ultraviolet radiation exposure activities. Int J Envion Res Public Health 2013;10:3203-16.

12. Thomas B, Fitzpatrick MD. The validity of sun reative skin types 1 through. Arch Dermatol 1988;6:869-71.

13. Diffey B. Sunscreen expectation and realisation. Photodermatol Photoimmunol Photomed 2009;25:233-6.

14. Baran R. $4^{\text {th }}$ edition. Floward Mali back Textbook of cosmetic, Dermatology; 2010.

15. Dutra EA, Oliveira DAGC, Hackmann ERMK, Santoro MIRM. Determination of sun protection factor (SPF) of sunscreens by ultraviolet spectrophotometry. Braz J Pharm Sci 2004;40:382-5.

16. Mishar AK, Mishar A, Chattopadhayay P. Assesment of in vitro sun protection factor of Calendalaofficinalis L. (Asteraceae) essential oil formulation. J Young Pharm 2012;4:17-21.

17. Azarbayjani AF, Tan PL, Chan SY. Ascorbic acid for the safe use of a sunscreen agent: accumulation of nano zinc oxide and titanium dioxide on the skin. Sci Pharm 2013;81:1141-50.

18. Young AR, Boles J, Herzog B, Osterwalder U, Baschong W. A sunscreen's labelled sun protection factor may overestimate protection at temperate latitudes: a human in vivo study. I Invest Dermatol 2010;130:2457-62.

19. Springsteen A, Yurek R, Carr MFKF. In vitro measurements of sun protection factor of sunscreens by diffuse transmittance. Anal Chim Acta 1999;380:155-64.

20. Australian Medical Handbook; 2011. p. 379-80.

21. Banks BA, Silerman RA, Schwartz RH, Tunnessen WW. Attitudes of teenagers towards sun exposure and sunscreen use. Pediatrics 1992;89:40-2.

22. Wright BPL, Green A. Do people who apply sunscreen, reapply them. Australian Journal of Dermatology; 2002.

23. Katzung BG, Masters SB, Trever AJ. Basic and clinical pharmacology. Chapter 61. Tata McGraw Hill Education Private Limited, New Delhi. 12 th edition; 2012. p. 1069.

24. Kaur CD, Saraf S. In vitro sun protection factor determination of herbal oils used in cosmetics. Pharmacogn Res 2010;2:22-5.

25. Rego D, Fernandes L, Nascimento T, Grenha A. Evaluation of sunscreen during a typical beach period. J Pharm BioAllied Sci 2010;2:47-50.

\section{How to cite this article}

- $\quad$ NGPD Nawarathna, HMDR Herath, DBM Wickramarathne, MHF Sakeena, CB Gunawardhane, SHT Sudeshika. Awareness of usage of sunscreens among school children in kandy, Sri Lanka. Int J Pharm Pharm Sci 2017;9(1):311-314. 